\title{
Potent small molecule extinguishes the NLRP3 inflammasome
}

An international team of researchers has uncovered "a highly potent and selective inhibitor of NLRP3 which could have utility as a research tool and possibly a therapeutic for many diseases," says Luke O'Neill, corresponding author (together with Matthew Cooper) of the Nature Medicine paper describing the findings. "Our work also confirms that in inflammation there are common processes which are relevant to multiple diseases - in this case NLRP3 activation."

The small molecule, known as MCC950, was first described by Pfizer in 2001 as an inhibitor of IL- $1 \beta$ processing. "However," as Cooper explains, "they did not know how the compounds worked, their target or mode of

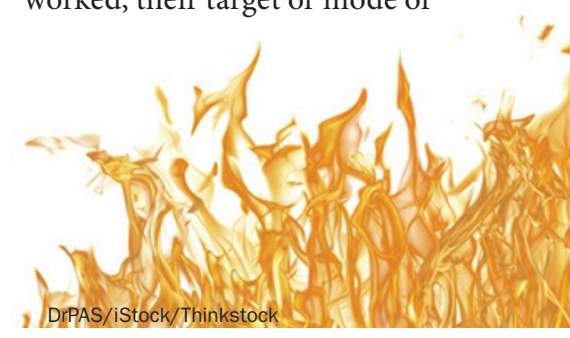

action." With this latest work, O'Neill, Cooper and their collaborators showed that MCC950 potently and specifically inhibits the NACHT, LRR and PYD domains-containing protein 3 (NLRP3) inflammasome, an intracellular complex that has been implicated in numerous autoinflammatory and autoimmune diseases.

In mouse and human macrophages, nanomolar concentrations of MCC950 inhibited NLRP3 inflammasome activation in response to canonical and noncanonical stimuli, but did not inhibit other inflammasome complexes, Toll-like receptor signalling or the

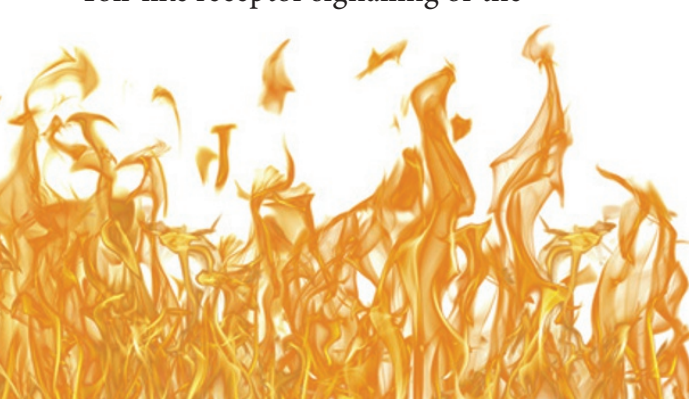

priming phase of NLRP3 activation. In animal models of NLRP3-mediated diseases, treatment with MCC950 attenuated the severity of experimental autoimmune encephalitis (a mouse model of human multiple sclerosis) and prevented neonatal death in a mouse model of Muckle-Wells syndrome (MWS), a cryopyrin-associated periodic syndrome caused by gain-of-function mutations in NLRP3. Finally, MCC950 was shown to be active and to inhibit NLRP3 activation in peripheral blood mononuclear cells from individuals with MWS ex vivo.

The investigators plan to further analyse the mechanism of action of MCC950 as well as its therapeutic potential in a range of diseases.

\section{Sarah Onuora}

Original article Coll, R. C. et al. A small-molecule inhibitor of the NLRP3 inflammasome for the treatment of inflammatory diseases. Nat. Med. doi:10.1038/ $\mathrm{nm} .3806$ 